Pacific Journal of Mathematics

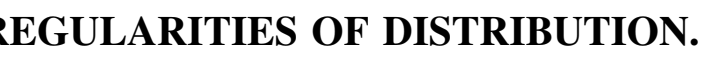




\section{IRREGULARITIES OF DISTRIBUTION III}

\section{Wolfgang M. SchmidT}

This paper deals with irregularities of distribution on spheres. Suppose there are $N$ points on the unit sphere $S=S^{n}$ of Euclidean $E^{n+1}$. If these points are reasonably well distributed one would expect that for every simple measurable subset $A$ of the sphere the number $\nu(A)$ of these points in the subset is fairly close to $N \mu(A)$, where $\mu$ denotes the measure which is normalized so that $\mu(S)=1$. Hence define the discrepancy $\Delta(A)$ by

$$
\Delta(A)=|\nu(A)-N \mu(A)| .
$$

It is shown in the present paper that there are very simple sets $A$, namely intersections of two half spheres, for which $\Delta(A)$ is large. This result is analogous to a theorem of $K$. F. Roth concerning irregularities of distribution in an $n$-dimensional cube.

To be more precise, let $\omega(\boldsymbol{x}, \boldsymbol{y})$ be the spherical distance of two points $\boldsymbol{x}, \boldsymbol{y}$ on $S$. If $\boldsymbol{x}$ is on $S$, let $H(\boldsymbol{x})$ be the half sphere consisting of points $z$ on $S$ with $\omega(\boldsymbol{x}, z) \leqq \pi / 2$, and if $\boldsymbol{x}$ and $\boldsymbol{y}$ are on $S$, let $L(\boldsymbol{x}, \boldsymbol{y})$ be the slice defined by

$$
L(\boldsymbol{x}, \boldsymbol{y})=H(\boldsymbol{x}) \cap H(\boldsymbol{y}) .
$$

Theorem 1. Suppose $n=2$. Then

$$
\int_{S} \int_{S} \Delta(L(\boldsymbol{x}, \boldsymbol{y}))^{2} d_{S} \boldsymbol{x} d d_{S} \boldsymbol{y} \geqq c_{1} \log N .
$$

Corollary. Again suppose that $n=2$. There are slices $L=$ $L(\boldsymbol{x}, \boldsymbol{y})$ with

$$
\Delta(L) \geqq c_{2}(\log N)^{1 / 2},
$$

and hence there are spherical triangles $T$ with

$$
\Delta(T) \geqq c_{3}(\log N)^{1 / 2} .
$$

ThEOREM 2. Suppose $n>2$. Then

$$
\int_{S} \int_{S} A(L(\boldsymbol{x}, \boldsymbol{y}))^{2} d_{S} \boldsymbol{x} d d_{S} \boldsymbol{y} \geqq c_{4}(n) N^{1-(2 / n)} .
$$

Corollary. Assume that $n>2$. There are slices $L=L(\boldsymbol{x}, \boldsymbol{y})$ with 


$$
\Delta(L) \geqq c_{5}(n) N^{(1 / 2)-(1 / n)} \text {. }
$$

As was mentioned above, our theorems are similar to a theorem of K. F. Roth [2]. In contrast to Roth we prove our theorems by means of certain integral equations. The method is entirely analogous to that used in the second paper of this series, although the details are rather simpler in the present case. Neither of the two previous papers [3], [4] of this series is prerequisite for reading the present paper.

P. Erdös asked about the existence of "spherical caps" $C$ on $S$ with $\Delta(C)$ large. At present we are unable to prove anything in this direction. In fact the proofs of our estimates for slices depend on the trivial estimate.

$$
\Delta(C) \geqq\|N \mu(C)\|
$$

for caps, where \|\| denotes the distance from the nearest integer. ${ }^{1}$

There are no known examples of $N$ points for which $\Delta(L)$ is small for all slices $L$. I believe it would be possible to construct a distribution of $N$ points with $\Delta(L) \ll N^{1-(1 / n)}$. Perhaps probabilistic methods would yield even better upper estimates.

2. An integral equation. For $0<\rho \leqq \pi / 2$ and for $z \in S$, let $C(\rho ; z)$ be the spherical cap of radius $\rho$ and center $z$ consisting of the points $\boldsymbol{x}$ with

$$
\omega(x, z) \leqq \rho
$$

and let $\mu(\rho)$ be the measure of a cap of radius $\rho$. Put

$$
f_{C}(\rho ; z \mid \boldsymbol{x})=\left\{\begin{array}{l}
1 \text { if } \boldsymbol{x} \in C(\rho ; \mathbf{z}), \\
0 \text { otherwise } .
\end{array}\right.
$$

Given two points $\boldsymbol{x}, \boldsymbol{y}$ on $S$ put

$$
h_{C}(\rho ; \boldsymbol{x}, \boldsymbol{y})=\int_{S} f_{C}(\rho ; \boldsymbol{z} \mid \boldsymbol{x}) f_{C}(\rho ; \boldsymbol{z} \mid \boldsymbol{y}) d_{S} z
$$

It is clear that $h_{c}(\rho ; \boldsymbol{x}, \boldsymbol{y})$ as a function of $\boldsymbol{x}, \boldsymbol{y}$ depends only on the spherical distance $\omega(\boldsymbol{x}, \boldsymbol{y})$ :

$$
h_{C}(\rho ; \boldsymbol{x}, \boldsymbol{y})=k_{C}(\rho ; \omega(\boldsymbol{x}, \boldsymbol{y}))
$$

Put

$$
f_{L}(z, \boldsymbol{w} \mid \boldsymbol{x})=\left\{\begin{array}{l}
1 \text { if } \boldsymbol{x} \in L(z, \boldsymbol{w}), \\
0 \text { otherwise },
\end{array}\right.
$$

and write

1 Added in proof. Recently (Irreg. of Distr. IV, Inventiones math 7 (1969), 55-82) I succeeded in proving results on caps. Combining these results with the integral equations of the present paper I obtained improved versions of Theorems 1 and 2 . 


$$
h_{L}(\boldsymbol{x}, \boldsymbol{y})=\int_{S} \int_{S} f_{L}(z, \boldsymbol{w} \mid \boldsymbol{x}) f_{L}(z, \boldsymbol{w} \mid \boldsymbol{y}) d_{S} z d_{S} \boldsymbol{w} .
$$

There is a function $k_{L}(\omega)$ such that

$$
h_{L}(\boldsymbol{x}, \boldsymbol{y})=k_{L}(\omega(\boldsymbol{x}, \boldsymbol{y})) \text {. }
$$

Lemma 1. Suppose $f(\rho)$ is nonnegative and continuous in $0<\rho \leqq \pi / 2$ and satisfies the integral equation

$$
\int_{0}^{\pi / 2} k_{C}(\rho ; \omega) f(\rho) d \rho=k_{L}(\omega) \quad(0 \leqq \omega \leqq \pi) .
$$

Then

$$
\int_{0}^{\pi / 2} d \rho \int_{S} d_{S} z \Delta(C(\rho ; z))^{2} f(\rho)=\int_{S} \int_{S} \Delta(L(z, \boldsymbol{w}))^{2} d_{S} z d_{S} \boldsymbol{w}
$$

Proof. We observe that

$$
\begin{aligned}
\int_{S} \int_{S} k_{C}(\rho ; \omega(\boldsymbol{x}, \boldsymbol{y})) d_{S} \boldsymbol{x} d d_{S} \boldsymbol{y} & =\int_{S} \int_{S} \int_{S} f_{C}(\rho ; \boldsymbol{z} \mid \boldsymbol{x}) f_{C}(\rho ; \boldsymbol{z} \mid \boldsymbol{y}) d_{S} \boldsymbol{x} d d_{S} \boldsymbol{y} d_{S} \boldsymbol{z} \\
& =\mu(\rho)^{2} .
\end{aligned}
$$

Let $\boldsymbol{p}_{1}, \cdots, \boldsymbol{p}_{N}$ be the given $N$ points on the sphere. We have

$$
\begin{aligned}
\int_{S} \Delta(C(\rho ; z))^{2} d_{S} z= & \sum_{i, j=1}^{N} \int_{S} f_{C}\left(\rho ; z \mid \boldsymbol{p}_{i}\right) f_{C}\left(\rho ; \boldsymbol{z} \mid \boldsymbol{p}_{j}\right) d_{S} z \\
& -2 N \mu(\rho) \sum_{i=1}^{N} \int_{S} f_{C}\left(\rho ; z \mid \boldsymbol{p}_{i}\right) d_{S} \boldsymbol{z}+N^{2} \mu(\rho)^{2} \\
= & \sum_{i . j=1}^{N}\left(k_{C}\left(\rho ; \omega\left(\boldsymbol{p}_{i}, \boldsymbol{p}_{j}\right)\right)-\mu(\rho)^{2}\right) \\
= & \sum_{i, j=1}^{N}\left(k_{C}\left(\rho ; \omega\left(\boldsymbol{p}_{i}, \boldsymbol{p}_{j}\right)\right)-\int_{S} \int_{S} k_{C}(\rho ; \omega(\boldsymbol{x}, \boldsymbol{y})) d_{S} \boldsymbol{x} d_{S} \boldsymbol{y}\right) .
\end{aligned}
$$

In a similar fashion one arrives at

$$
\begin{aligned}
& \int_{S} \int_{S} L(L(z, \boldsymbol{w}))^{2} d_{S} z d_{S} \boldsymbol{w} \\
& \quad=\sum_{i, j=1}^{N}\left(k_{L}\left(\omega\left(\boldsymbol{p}_{i}, \boldsymbol{p}_{j}\right)\right)-\int_{S} \int_{S} k_{L}(\omega(\boldsymbol{x}, \boldsymbol{y})) d_{S} \boldsymbol{x} d_{S} \boldsymbol{y}\right) .
\end{aligned}
$$

The equation (14) now is an immediate consequence of (13), (15) and (16).

3. Application of the integral equation. Assume that $n \geqq 2$. In $\S 5$ we shall find a continuous nonnegative solution $f(\rho)$ of (13) which satisfies

$$
f(\rho) \gg \rho^{1-n}
$$


as $\rho \rightarrow 0$. Hence by virtue of (8) the left hand side and therefore also the right hand side of (14) exceeds

$$
c_{6}(n) \int_{0}^{c_{7}(n)} \rho^{1-n}\|N \mu(\rho)\|^{2} d \rho .
$$

Here

$$
\mu(\rho)=c_{8}(n) \int_{0}^{\rho}(\sin \varphi)^{n-1} d \varphi=c_{9}(n) \rho^{n}+c_{9,1}(n) \rho^{n+1}+\cdots .
$$

To evaluate (18) we put

$$
x=N \mu(\rho)=N c_{9}(n) \rho^{n}+N c_{9,1}(n) \rho^{n+1}+\cdots .
$$

We obtain the lower bound

$$
c_{10}(n) N^{1-(2 / n)} \int_{1}^{c_{11}(n) N} x^{(2 / n)-2} d x .
$$

for (18). When $n=2$ this is

$$
\geqq c_{1}(n) \log N
$$

and Theorem 1 follows. When $n>2$ then (21) is

$$
\geqq c_{4}(n) N^{1-(2 / n)}
$$

and Theorem 2 follows.

4. Auxiliary functions.

LEMMA 2.

$$
k_{L}(\omega)=\left(\frac{1}{2 \pi}(\pi-\omega)\right)^{2} .
$$

Proof. Suppose $\boldsymbol{x}, \boldsymbol{y}$ are any two points on $S$ with $\omega(\boldsymbol{x}, \boldsymbol{y})=\omega$. Then we have

$$
\begin{aligned}
k_{L}(\omega) & =h_{L}(\boldsymbol{x}, \boldsymbol{y})=\int_{S} \int_{S} f_{L}(z, \boldsymbol{w} \mid \boldsymbol{x}) f_{L}(z, \boldsymbol{w} \mid \boldsymbol{y}) d_{S} z d_{S} \boldsymbol{w} \\
& =\int_{S} \int_{S} f_{L}(\boldsymbol{x}, \boldsymbol{y} \mid \boldsymbol{z}) f_{L}(\boldsymbol{x}, \boldsymbol{y} \mid \boldsymbol{w}) d_{S} z d_{S} \boldsymbol{w}=(\mu(L(\boldsymbol{x}, \boldsymbol{y})))^{2} \\
& =\left(\frac{1}{2 \pi}(\pi-\omega(\boldsymbol{x}, \boldsymbol{y}))\right)^{2} .
\end{aligned}
$$

We say a function $f(y)$ defined in $0<y \leqq \pi$ is of the type $(r, s)$ where $r, s$ are nonnegative integers if it can be written as a finite sum of the type 


$$
\sum_{i=1}^{b} a_{i}(\cos (y / 2))^{r_{i}}(\sin (y / 2))^{-s_{i}}
$$

where the coefficients $\alpha_{i}$ are positive and $r_{i}, s_{i}$ are integers with

$$
r_{i} \geqq r(i=1, \cdots, b), s_{i} \geqq 1(i=1, \cdots, b) \text { and } \max \left(s_{1}, \cdots, s_{b}\right)=s .
$$

We say $f(y)$ is of the type $(r, s)^{\prime}$ if it satisfies the same conditions as before except that some of the exponents $s_{i}$ may be 0 rather than $\geqq 1$ as in (22).

If $r>0, s>0$ and $f(y)$ is of the type $(r, s)$, then $-f^{\prime}(y)$ is of the type $(r-1, s+1)^{\prime}$ and $-f^{\prime}(y) \cos ^{3}(y / 2)(\sin (y / 2))^{-1}$ is of the type $(r+2, s+2)$.

We are going to construct functions $l_{1}(y), l_{2}(y), \cdots, l_{t}(y)$ where

$$
t=\{(n+1) / 2\}
$$

i.e., the smallest integer $t \geqq(n+1) / 2$, as follows. Our functions will be defined in $0<y \leqq \pi$. First put

$$
l_{1}(y)=-2 \pi k_{L}^{\prime}(y)=2(\pi-y)
$$

and

$$
\begin{aligned}
l_{2}(y) & =-2(n-1)^{-1} \cos ^{3}(y / 2)(\sin y / 2)^{-1} l_{1}^{\prime}(y) \\
& =4(n-1)^{-1} \cos ^{3}(y / 2)(\sin (y / 2))^{-1} .
\end{aligned}
$$

Then $l_{2}(y)$ is of the type $(3,1)$.

Now suppose $t>2$ and $l_{j-1}(y)$ with $3 \leqq j \leqq t$ has already been constructed and is of the type $(2(j-1)-1,2(j-1)-3)$. Then put

$$
l_{j}(y)=-2(n+3-2 j)^{-1} \cos ^{3}(y / 2)(\sin (y / 2))^{-1} l_{j-1}^{\prime}(y) .
$$

Then $l_{j}(y)$ is of the type $(2 j-1,2 j-3)$.

This finishes our construction of $l_{1}(y), \cdots, l_{t}(y)$. In particular, $l_{t}(y)$ is of the type $(2 t-1,2 t-3)$ and $-l_{t}^{\prime}(y)$ is of the type $(2 t-2,2 t-2)^{\prime}$.

Lemma 3. Suppose $0<\rho \leqq \pi / 2$ and $0 \leqq \omega \leqq \pi$. Then

$$
k_{c}(\rho ; \omega)=(n-1) \pi^{-1} \int_{0}^{c(\rho, \omega / 2)}(c(\rho, \varphi)-(\omega / 2)) \cos \varphi(\sin \varphi)^{n-2} d \varphi
$$

where

$$
c(x, y)=\left\{\begin{array}{l}
\operatorname{arc} \cos (\cos x / \cos y) \text { if } 0 \leqq y<x \leqq \pi / 2 \\
0 \text { otherwise }
\end{array}\right.
$$

Proof. The function $k_{C}(\rho ; \omega)$ is equal to the measure of the 
intersection of two spherical caps of radius $\rho$ whose centers have spherical distance $\omega$. It is therefore zero when $\omega \geqq 2 \rho$, and since also $c(\rho, \omega / 2)$ is zero in this case, the formula (25) is then correct.

Hence assume that $0 \leqq \omega<2 \rho$. We may write

$$
k_{c}(\rho ; \omega)=h_{c}(\rho ; \boldsymbol{x}, \boldsymbol{y})
$$

where $\boldsymbol{x}, \boldsymbol{y}$ are any two points on $S$ having $\omega(\boldsymbol{w}, \boldsymbol{y})=\omega$. We choose

$$
\begin{aligned}
& \boldsymbol{x}=(\cos (\omega / 2), \sin (\omega / 2), 0, \cdots, 0), \\
& \boldsymbol{y}=(\cos (\omega / 2),-\sin (\omega / 2), 0, \cdots, 0) .
\end{aligned}
$$

Every point $z$ on $S^{n}$ with $n \geqq 2$ may be written

$$
z=(\cos \varphi \cos \psi, \cos \varphi \sin \psi,(\sin \varphi) \boldsymbol{w})
$$

where $0 \leqq \varphi \leqq \pi / 2, \quad 0 \leqq \psi<2 \pi$ and $w$ is on $S^{n-2}$. This representation is unique except for a set of measure zero, and

$$
d_{S} z=(2 \pi)^{-1}(n-1) \cos \varphi(\sin \varphi)^{n-2} d \varphi d \psi d d_{S^{n-2}} \boldsymbol{w} .
$$

(When $n=2$ then $S^{n-2}$ consists of only two points $(1)$ and $(-1)$ and the integral of a function $f(\boldsymbol{w})$ over $S^{n-2}$ is $(1 / 2)(f(1)+f(-1))$ ).

One has $h_{c}(\rho ; \boldsymbol{x}, \boldsymbol{y})=\mu(C(\rho, \boldsymbol{x}) \cap C(\rho ; \boldsymbol{y}))$. The point $z$ lies in $C(\rho ; \boldsymbol{x})$ and in $C(\rho ; \boldsymbol{y})$ if and only if the point

$$
z^{\prime}=(\cos \varphi \cos \psi, \cos \varphi \sin \psi, \sin \varphi)
$$

satisfies

$$
\omega\left(z^{\prime}, x^{\prime}\right) \leqq \rho \text { and } \omega\left(z^{\prime}, y^{\prime}\right) \leqq \rho
$$

with $x^{\prime}=(\cos (\omega / 2), \sin (\omega / 2), 0)$ and $y^{\prime}=(\cos (\omega / 2),-\sin (\omega / 2), 0)$. We are thus left with a problem on the 2 -sphere.

By symmetry it will suffice to look at points $z^{\prime}$ with $0 \leqq \psi \leqq \pi$, and for such points $z^{\prime}$ one has $\omega\left(z^{\prime}, x^{\prime}\right) \leqq \omega\left(z^{\prime}, y^{\prime}\right)$. The points $\boldsymbol{y}^{\prime}, \boldsymbol{z}^{\prime}, \boldsymbol{u}^{\prime}=(\cos \psi, \sin \psi, 0)$ form a spherical triangle with a right angle at $\boldsymbol{u}^{\prime}$. Hence by a formula in spherical trigonometry,

$$
\cos \left(\omega\left(z^{\prime}, \boldsymbol{y}^{\prime}\right)\right)=\cos \left(\omega\left(z^{\prime}, \boldsymbol{u}^{\prime}\right)\right) \cos \left(\omega\left(\boldsymbol{y}^{\prime}, \boldsymbol{u}^{\prime}\right)\right)=\cos \varphi \cos (\psi+(\omega / 2)),
$$

and $\omega\left(z^{\prime}, \boldsymbol{y}^{\prime}\right) \leqq \rho$ is true exactly if

$$
\cos \varphi \cos (\psi+(\omega / 2)) \geqq \cos \rho .
$$

Since we have $0 \leqq \psi \leqq \pi$ and $0 \leqq \omega<\pi$, this implies $\cos \varphi \cos (\omega / 2) \geqq$ $\cos \rho$ and therefore

$$
0 \leqq \varphi \leqq c(\rho, \omega / 2)
$$

If $\varphi$ satisfies $0 \leqq \varphi<c(\rho, \omega / 2)$, then $\psi$ is in the interval 


$$
0 \leqq \psi \leqq c(\rho, \phi)-(\omega / 2)
$$

Combining the information gathered we obtain

$$
k_{C}(\rho ; \omega)=2(2 \pi)^{-1}(n-1) \int_{0}^{c(\rho, \omega / 2)} d \varphi \cos \varphi(\sin \varphi)^{n-2} \int_{0}^{c(\rho, \varphi)-(\omega / 2)} d \psi \int_{S^{n-2}} d_{S} \boldsymbol{w},
$$

and (25) follows.

Corollary. Suppose $0<\omega<2 \rho<\pi$. Then

$$
\frac{\partial}{\partial \omega} k_{C}(\rho ; \omega)=-(2 \pi)^{-1}\left(1-\left(\cos ^{2} \rho / \cos ^{2}(\omega / 2)\right)\right)^{(n-1) / 2} .
$$

Proof. Using the standard rules for differentiation of an integral (for a justification see, e.g., [4, Lemma 2]), we derive from (25) the equation

$$
\begin{aligned}
\frac{\partial}{\partial \omega} k_{C}(\rho ; \omega) & =-(n-1)(2 \pi)^{-1} \int_{0}^{c(\rho, \omega / 2)} \cos \varphi(\sin \varphi)^{n-2} d \varphi \\
& =-(2 \pi)^{-1}(\sin (c(\rho, \omega / 2)))^{n-1} \\
& =-(2 \pi)^{-1}\left(1-\left(\cos ^{2} \rho / \cos ^{2}(\omega / 2)\right)\right)^{(n-1) / 2} .
\end{aligned}
$$

5. Solution of the integral equation. Suppose $f(\rho)$ is continuous in $0<\rho \leqq \pi$ and satisfies

$$
f(\rho) \ll \rho^{1-n}
$$

as $\rho \rightarrow 0$. Since

$$
k_{c}(\rho ; \omega) \leqq \mu(\rho) \ll \rho^{n}
$$

by (19), the function $k_{c}(\rho ; \omega) f(\rho)$ tends uniformly to zero as $\rho \rightarrow 0$, and hence may be extended to a function which is continuous in $0 \leqq \rho \leqq \pi / 2,0 \leqq \omega \leqq \pi$. Therefore both sides of (13) are continuous functions of $\omega$, and it will suffice to show that $f(\rho)$ satisfies (13) in $0<\omega<\pi$. The equation (13) may be rewritten as

$$
\int_{\omega / 2}^{\pi / 2} k_{c}(\rho ; \omega) f(\rho) d \rho=k_{L}(\omega) \quad(0<\omega<\pi) .
$$

Differentiating and using the corollary to Lemma 3 we obtain

$$
-(2 \pi)^{-1} \int_{\omega / 2}^{\pi / 2}\left(1-\left(\cos ^{2} \rho / \cos ^{2}(\omega / 2)\right)\right)^{(n-1) / 2} f(\rho) d \rho=k_{L}^{\prime}(\omega) \quad(0<\omega<\pi) .
$$

Since both sides of (33) tend to zero as $\omega \rightarrow \pi$, any solution of (34) is also a solution of (33). We now change the notation and rewrite (34) in the form 


$$
\int_{y / 2}^{\pi / 2} f(x)\left(1-\left(\cos ^{2} x / \cos ^{2}(y / 2)\right)^{(n-1) / 2} d x=l_{1}(y) \quad(0<y<\pi) .\right.
$$

Differentiating again and simplifying we obtain

$$
\int_{y / 2}^{\pi / 2} \cos ^{2} x f(x)\left(1-\left(\cos ^{2} x / \cos ^{2}(\mathrm{y} / 2)\right)\right)^{(n-3) / 2} d x=l_{2}(y) \quad(0<y<\pi) .
$$

and any solution of this equation is in fact also a solution of (35). We may continue in this fashion until we arrive at the equation

$$
\int_{y / 2}^{\pi / 2}(\cos x)^{2 t-2} f(x)\left(1-\left(\cos ^{2} x / \cos ^{2}(y / 2)\right)\right)^{(n+1-2 t) / 2} d x=l_{t}(y) \quad(0<y<\pi) .
$$

We now have to distinguish the cases when $n$ is even and when $n$ is odd. First assume that

$$
n \text { is odd }
$$

Then $t=(n+1) / 2$ and (36) reduces to

$$
\int_{y / 2}^{\pi / 2}(\cos x)^{n-1} f(x) d x=l_{t}(y) \quad(0<y<\pi) .
$$

Differentiating once more we obtain

$$
-\frac{1}{2}(\cos (y / 2))^{n-1} f(y / 2)=l_{t}^{\prime}(y) \quad(0<y<\pi)
$$

whence

$$
f(x)=-2 l_{t}^{\prime}(2 x)(\cos x)^{1-n} \quad(0<x<\pi / 2) .
$$

Since both sides of (37) tend to zero as $y \rightarrow \pi$, this is in fact a solution of (37).

The function

$$
f(y / 2)=-2 l_{t}^{\prime}(y)(\cos (y / 2))^{1-n} \quad(0<y<\pi)
$$

is the restriction to $0<y<\pi$ of a function of the type

$$
(2 t-2+1-n, 2 t-2)^{\prime}=(0, n-1)^{\prime} .
$$

It follows that $f(x)$ is positive in $0<x<\pi / 2$ and it may be extended to a function which is continuous in $0<x \leqq \pi / 2$. Finally, when $x$ is small then $f(x)$ has the same order of magnitude as $(\sin x)^{1-n}$, and we have

$$
x^{1-n} \ll f(x) \ll x^{1-n} .
$$

Thus (17) and (32) are satisfied.

Now assume that 


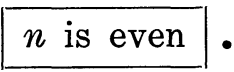

Then $t=(n+2) / 2$ and (36) may be rewritten as

(39) $\int_{y / 2}^{\pi / 2}(\cos x)^{n} f(x)\left(\sin ^{2} x-\sin ^{2}(y / 2)\right)^{-1 / 2} d x=l_{t}(y) / \cos (y / 2) \quad(0<y<\pi)$.

The right hand side of this equation is the restriction to $0<y<\pi$ of some function $m(y)$ of the type $(2 t-2,2 t-3)=(n, n-1)$.

The substitution $\sin ^{2} x=X, \sin ^{2}(y / 2)=Y$ transforms (39) into an integral equation of Abel type which can be readily solved ([1], Chapter I). One obtains the solution

$$
\begin{aligned}
f(x)=-2 \pi^{-1} \sin x(\cos x)^{1-n} \int_{2 x}^{\pi} m^{\prime}(t)\left(\sin ^{2}(t / 2)-\sin ^{2} x\right)^{-1 / 2} d t & \\
& (0<x<\pi / 2) .
\end{aligned}
$$

Indeed, if we substitute this expression for $f(x)$ into the left hand side of (39) we obtain

$$
\begin{aligned}
& -2 \pi^{-1} \int_{y / 2}^{\pi / 2} d x \sin x \cos x\left(\sin ^{2} x-\sin ^{2}(y / 2)\right)^{-1 / 2} \\
& \times \int_{2 x}^{\pi} d t m^{\prime}(t)\left(\sin ^{2}(t / 2)-\sin ^{2} x\right)^{-1 / 2} \\
= & \pi^{-1} \int_{y}^{\pi} d t m^{\prime}(t) \int_{y / 2}^{t / 2} d x 2 \sin x \cos x\left(\sin ^{2} x-\sin ^{2}(y / 2)\right)^{-1 / 2} \\
& \times\left(\sin ^{2}(t / 2)-\sin ^{2} x\right)^{-1 / 2}
\end{aligned}
$$

The inner integral is equal to $\pi$ and we get

$$
-\int_{y}^{1} m^{\prime}(t) d t=m(y)-m(\pi)=m(y)
$$

as desired.

The function $-m^{\prime}(y)$ is of the type $(n-1, n)^{\prime}$ and hence we have

$$
\left.-m^{\prime}(t)=\cos (t / 2)\right)^{n-1}(\sin (t / 2))^{-n} l(t)
$$

where $l(t)$ is a continuous function in $0 \leqq t \leqq \pi$ with $l(0)>0$. Now $\sin (t / 2)$ and its inverse function are continuous in $0 \leqq t \leqq \pi$, and we may therefore write

$$
l(t)=L(\sin (t / 2))
$$

where $L(T)$ is a continuous function in $0 \leqq T \leqq 1$ which has $L(0)>0$. Substituting (41) into (40) and putting $\sin (t / 2)=T$ we obtain 


$$
\begin{aligned}
f(x)= & 4 \pi^{-1} \sin x(\cos x)^{1-n} \int_{\sin x}^{1} L(T) T^{-n}\left(1-T^{2}\right)^{(n-2) / 2} \\
& \times\left(T^{2}-\sin ^{2} x\right)^{-1 / 2} d T \quad\left(0<x<\frac{\pi}{2}\right) .
\end{aligned}
$$

Now $l(t)$ is positive in $0<t<\pi$ and hence $f(x)$ is positive and continuous in $0<x<\pi / 2$. One has

$$
\lim _{x \rightarrow \pi / 2} f(x)=4 \pi^{-1} L(1) \lim _{x \rightarrow \pi / 2}(\cos x)^{1-n} \int_{\sin x}^{1}\left(1-T^{2}\right)^{(n-2) / 2}\left(T^{2}-\sin ^{2} x\right)^{-1 / 2} T d T,
$$

provided the limit on the right hand side exists. But the expression to the right of lim on the right hand side is in fact a constant, and therefore the limit does exist. It follows that $f(x)$ may be extended to a function which is continuous in $0<x \leqq \pi / 2$.

Putting $T=u \sin x$ we obtain from (42) the estimate

$$
f(x) \ll x \int_{1}^{1 / \sin x}(\sin x)^{-n} u^{-n}\left(u^{2}-1\right)^{-1 / 2} d u \ll x^{1-n}
$$

as $x \rightarrow 0$. Therefore (32) holds. When $0<T<c_{12}(n)$, then $L(T) \gg 1$. It follows that

$$
f(x) \gg x \int_{1}^{c_{12}(n) / \sin x}(\sin x)^{-n} u^{-n}\left(u^{2}-1\right)^{-1 / 2} d u \gg x^{1-n}
$$

when $x$ is small, and (17) is satisfied.

\section{REFERENCES}

1. G. Kowalewski, Integralgleichungen, Walter de Gruyter and Co., Berlin and Leipzig, 1930.

2. K. F. Roth, On irregularities of distribution, Mathematika 1 (1954), 73-79.

3. W. M. Schmidt, Irregularities of distribution, Quart, J. Math. (Oxford) (2) 19 (1968), 181-191.

4. - Irregularities of distribution II. Trans. Amer. Math. Soc. 136 (1969), $347-360$.

Received December 5, 1967.

UNIVERSITY OF COLORADO 



\section{Pacific Journal of Mathematics \\ Vol. 29, No. 1 \\ May, 1969}

Jorge Alvarez de Araya, A Radon-Nikodým theorem for vector and operator

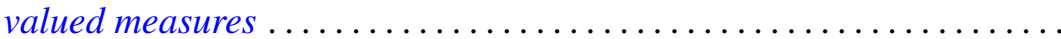

Deane Eugene Arganbright, The power-commutator structure of finite

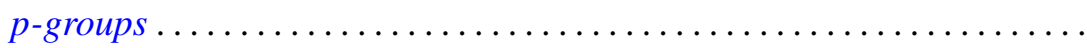

Richard Eugene Barlow, Albert W. Marshall and Frank Proschan, Some

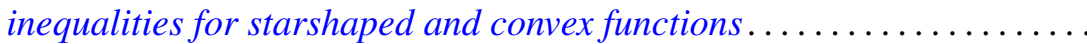

David Clarence Barnes, Some isoperimetric inequalities for the eigenvalues

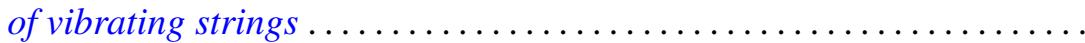

David Hilding Carlson, Critical points on rim-compact spaces ...........

Allan Matlock Weber Carstens, The lattice of pretopologies on an arbitrary

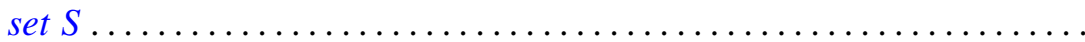

S. K. Chatterjea, A bilateral generating function for the ultraspherical

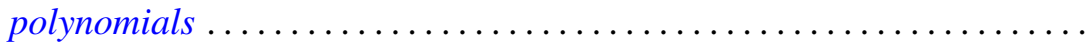

Ronald J. Ensey, Primary Abelian groups modulo finite groups ......... 77

Harley M. Flanders, Relations on minimal hypersurfaces ............ 83

Allen Roy Freedman, On asymptotic density in n-dimensions........... 95

Kent Ralph Fuller, On indecomposable injectives over artinian rings...... 115

George Isaac Glauberman, Normalizers of p-subgroups in finite groups . . . 137

William James Heinzer, On Krull overrings of an affine ring ........... 145

John McCormick Irwin and Takashi Ito, A quasi-decomposable abelian group without proper isomorphic quotient groups and proper isomorphic subgroups.

Allan Morton Krall, Boundary value problems with interior point boundary conditions

John S. Lowndes, Triple series equations involving Laguerre

polynomials

Philip Olin, Indefinability in the arithmetic isolic integers

Ki-Choul Oum, Bounds for the number of deficient values of entire functions whose zeros have angular densities..

R. D. Schafer, Standard algebras ....................

Wolfgang M. Schmidt, Irregularities of distribution. III.

Richard Alfred Tapia, An application of a Newton-like method to the Euler-Lagrange equation 